\title{
Multi-code Multicarrier CDMA: Performance Analysis
}

\author{
Taeyoon Kim, Jeffrey G. Andrews, Jaeweon Kim, and Theodore S. Rappaport
}

\begin{abstract}
A new multi-code multicarrier code division multiple access (MC-MC-CDMA) system is proposed and analyzed in a frequency selective fading channel. By allowing each user to transmit an $M$-ary code sequence, the proposed MC-MC-CDMA system can support various data rates as required by next generation standards without increasing the interference which is common in general multicarrier CDMA systems. The bit error rate of the system is analytically derived in frequency selective fading, with Gaussian noise and multiple access interference. The results show that the proposed MC-MC-CDMA system clearly outperforms both single-code multicarrier CDMA (MC-CDMA) and single-carrier multi-code CDMA in a fixed bandwidth allocation. This indicates that MC-MC-CDMA can be considered for next generation cellular systems.
\end{abstract}

Index Terms - multi-code, multicarrier CDMA, code division multiple access (CDMA), 4G cellular, time-frequency two dimensional gain

\section{INTRODUCTION}

$\mathrm{F}$ UTURE wireless systems such as fourth generation (4G) cellular will need flexibility to provide subscribers with a variety of services such as voice, data, images, and video. Because these services have widely different data rates and traffic profiles, future generation systems will have to accommodate a wide variety of data rates. Code division multiple access (CDMA) has proven very successful for large scale cellular voice systems, but there is some skepticism about whether CDMA will be well-suited to non-voice traffic [1]. This has motivated research on multi-code CDMA systems which allow variable data rates [2], [3], [4] by allocating multiple codes, and hence varying degrees of capacity to different users. Meanwhile, multicarrier CDMA (MC-CDMA) has emerged as a powerful alternative to conventional direct sequence CDMA (DS- CDMA) in mobile wireless communications [5], [6], [7], [8], [9], [10], and has been shown to have superior performance to single carrier CDMA in multipath fading. This paper proposes and analyzes a new multiple access and modulation technique: a combined multi-code multicarrier CDMA system for exploiting the best aspects of each of these earlier systems.

Manuscript received November 18, 2005; revised December 03, 2005, and February 25, 2006. This paper was presented in part at the IEEE International Conference on Communications (ICC'2004), Paris, France, June 2004.

The authors are with the Wireless Networking and Communications Group in the Department of Electrical and Computer Engineering at The University of Texas at Austin, Austin, TX 78712 USA. (e-mail: \{tykim, jandrews, jaeweon\}@ece.utexas.edu, wireless@mail.utexas.edu).
Multi-rate transmission for single-carrier CDMA systems in AWGN channels has been previously considered in the literature, e.g. [11], [12]. It is also an important part on third generation cellular standards, namely CDMA2000 1xEV-DO [13] and 1xEV-DV [14], known sometimes as HDR [15]. HDR supports diverse data rates using many codes with different spreading factors. However, in this case, the code assignment is limited by the number of orthogonal codes for the short spreading factor, and multipath can be very problematic for the higher data rates since the spreading factor is short. Unlike the HDR system, our proposed system does not require variable spreading factors. It uses the same code book to support various data rates for different users.

Multi-code techniques such as our proposed system tradeoff the number of supportable subscribers with the per subscriber data rate. Said another way, the number of simultaneous higher data rate users in a multi-code CDMA system will be less than the number of equal data rate users in a traditional CDMA system. A variation of the multi-code scheme, which supports variable data rates by varying the set of code sequences assigned to each of the users, has been proposed in [4], [16]. The users communicate their data by choosing one sequence from their code set to transmit over the common channel. Also, in [16] the performance of multi-code CDMA was considered only in an AWGN channel.

There have been to our knowledge two previous studies on multi-rate transmission for multicarrier direct sequence CDMA (MC-DS-CDMA) systems [17], [18]. The study of multi-rate transmission for MC-DS-CDMA systems based on the concepts of multi-code access and variable-spreading gain code access was first presented in [17]. In multi-rate MC-DS-CDMA, the data stream of a user with data rate $M$ is first multiplexed into $M$ different serial streams with a base data rate, and each serial stream is treated as an individual user. Each of the $M$ serial streams is then converted into $P$ parallel sub-streams and spread by the same spreading code with a constant spreading factor. Moreover, the system in [17] has $M$ times more interference per user, because each of the $M$ data streams is treated as an independent user. Therefore, the system of [17] experiences more interference as the data rate increases, even with a fixed number of users. Also, multi-rate transmission for frequency spread multicarrier CDMA has been studied in [19]. In the multi-rate multicarrier CDMA system in [19], the subcarriers are divided into $M$ groups according to the required data rate. Therefore, when the number of subcarrier is fixed, the spreading gain in frequency domain for each data is decreased with increasing data rate. The single-carrier multi-code CDMA 


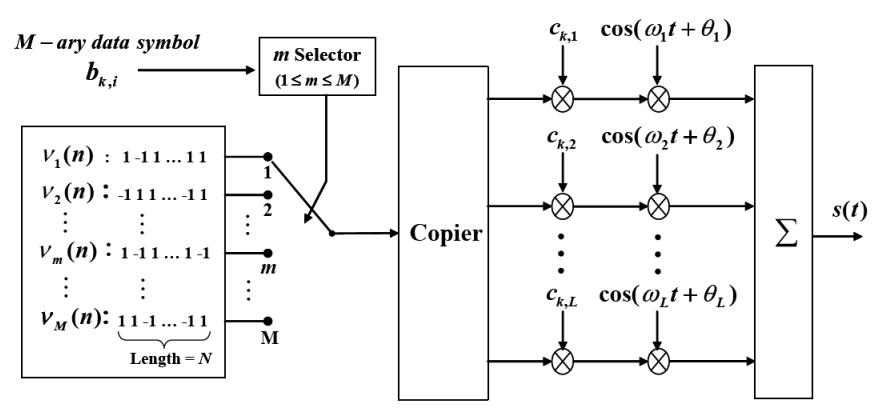

(a) Transmitter

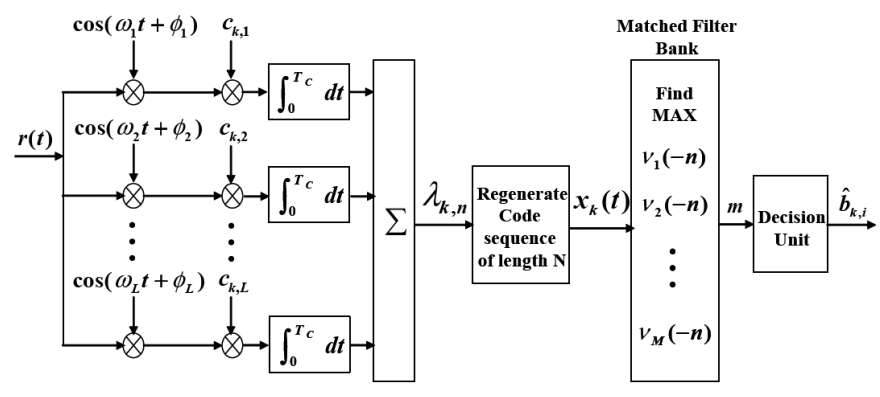

(b) Receiver

Fig. 1. Transmitter and receiver structure of MC-MC-CDMA

system in [4] addresses this interference scaling problem by using just one code sequence instead of spreading each of the $M$ multiplexed data streams so that the interference does not increase linearly with the data rate. However, this system [4] does not achieve the frequency diversity benefits of multicarrier modulation.

This contribution proposes a multicarrier CDMA system with multi-code that outperforms both [17] and [4]. Our proposed multi-code multicarrier CDMA (MC-MC-CDMA) system achieves the advantages of both systems: (i) variable data rates without interference scaling and (ii) enhanced robustness to multipath fading channels. Moreover, the proposed system has gain from both time and frequency domain to exploit the diversity and interference averaging properties of multicarrier modulation and CDMA.

The bit error probability of the proposed system is derived analytically and the improvement of the proposed system over an MC-CDMA system is shown through both analysis and simulations in a frequency selective fading channel. The rest of the paper is organized as follows: Section II discusses the system model and the characteristic of the gain of our proposed multi-code multicarrier CDMA system in the time and frequency domain. The bit error probability of the proposed system is derived in Section III, with a performance comparison presented in Section IV.

\section{Proposed SYSTEM}

\section{A. System Model}

The proposed MC-MC-CDMA system depicted in Fig. 1 uses a set of $M$ codes called the code sequence set for $M$-ary modulation. Each user has the same code sequence set which represents an information data symbol of $\log _{2} M$ bits. The size of the code sequence set depends on the required data rate. In the usual CDMA case, the size of the code sequence set is 2, i.e. there are two sequences in the set, one to represent a ' 0 ' and the other to represent a ' 1 '. In the proposed system, each user has a set of $M$ code sequences, where $\log _{2} M$ is the ratio of the required data rate to the base data rate (1 bit/symbol). Therefore, if the data rate is to be made $\log _{2} M$ times the base data rate, the size of the code sequence set is $M$ and each $M$-ary data symbol is mapped to one of the code sequences of length $N$. This code length $N$ is fixed over all different values of $M$. Thus, varying the data rate does not change the code length $N$, but it does change the size of the code sequence set $M$. If orthogonal code sequences are used, the performance advantages of orthogonal modulation are attained. However, in order to maintain linear independence between the code sets, it is required that $M \leq N$. If non-orthogonal code sequences are used, then $M$ can be greater than $N$, naturally at the expense of the distance between code symbols.

As shown in Fig. 1(a), an $M$-ary symbol selects one of $M$ pre-mapped code sequences for transmission. Each code sequence has a time domain length of $N$. Each bit of the length $N$ code sequence is copied onto the $L$ subcarrier branches and multiplied with the user-specific scrambling code of the corresponding branch, $c_{k, l}$. Note that the $c_{k, l}$ are independent of time so that the spreading at this stage is only in frequency, allowing users to choose specific codes that have low crosscorrelations with other user's codes. Each of these branches then modulates one of the $L$ orthogonal subcarriers and the results are summed. As in popular orthogonal frequency division multiplexing (OFDM), this process can be implemented using a size $L$ Inverse Fast Fourier Transform (IFFT) to replace the subcarrier multiplication and summation. Unlike OFDM, which uses serial to parallel conversion, in multicarrier CDMA the same information bit is replicated on all subcarriers to achieve a spreading gain for multiple access. Also, a cyclic prefix is not typically employed in multicarrier CDMA because self-ISI is a minor effect compared to multiple access interference.

\section{B. Time and Frequency Signaling Gain: Orthogonality and Spreading}

A multicarrier CDMA system with spreading only in the frequency domain is generally referred to as an MC-CDMA system, while a multicarrier system with spreading only in the time domain is usually called MC-DS-CDMA. As shown in Section II-A and Fig. 1, the proposed MC-MC-CDMA system has two-dimensional gain in both the time and frequency domains by using a multi-code signal and multicarrier modulation, respectively. Two-dimensional gain exploits both time and frequency domains and thus can simultaneously combat frequency selective fading and multiple-access interference (MAI) from the advantages of multicarrier modulation and CDMA.

The total gain with two-dimensional signaling is the product of the time domain gain which comes from the orthogonality between code sequences and the frequency spreading gain. Within a fixed total bandwidth, the two-dimensional gain can 
be adapted to the user load and radio link conditions such as Doppler spread, delay spread, and channel gain.

It may be noted that two-dimensional gain in the time and frequency domain is similar in principle to the MC-DS-CDMA system recently proposed in [10]. By using a frequency spreading code sequence, both systems can distinguish users while assigning the same time domain spreading codes to each user. MC-MC-CDMA improves upon MC-DS-CDMA in its handling of variable rates, and more efficient spreading codes. The latter property is due to the selection of one of $\mathrm{M}$ information-bearing codewords rather than multiplying a fixed codeword by the incoming data bit.

\section{System ANALYSIS}

\section{A. Signal Description}

To formalize the analysis of the MC-MC-CDMA system, consider that each user has the same code sequence set which can be written as

$$
\Omega=\left\{v_{m}(n) \mid 1 \leq m \leq M\right\} .
$$

User $k$ 's $i^{\text {th }} M$-ary data symbol $b_{k, i}$ is mapped to one of the code sequences in $\Omega$. Thus, the $i^{\text {th }}$ transmit sequence of user $k$ before multicarrier modulation can be written as

$$
\begin{gathered}
S_{k, i}(n)=v_{b_{k, i}}(n), \\
S_{k, i}(t)=\sum_{n=0}^{N-1} S_{k, i}(n) h\left(t-n T_{c}-i T_{s}\right),
\end{gathered}
$$

where $S_{k, i}(n)$ is the $n^{\text {th }}$ bit in the $i^{\text {th }}$ code sequence of user $k, T_{c}$ is the bit duration of the code sequence, $T_{s}$ is the symbol duration $\left(T_{s}=N T_{c}\right), N$ is the length of code sequence, and $h(t)$ is the normalized rectangular waveform defined as

$$
h(t)= \begin{cases}\frac{1}{\sqrt{T_{c}}} & , \quad 0 \leq t \leq T_{c} \\ 0 & , \text { elsewhere }\end{cases}
$$

According to the block diagram of the MC-MC-CDMA transmitter shown in Fig. 1(a) and (4), the transmitted BPSK signal of user $k$ can be written as

$$
\begin{aligned}
s_{k}(t)=\sum_{i=-\infty}^{\infty} \sum_{l=1}^{L} \sum_{n=0}^{N-1} S_{k, i}(n) h( & \left.t-n T_{c}-i T_{s}\right) \\
& \times c_{k, l}(N i+n) \cos \left(\omega_{l} t+\theta_{k, l}\right),
\end{aligned}
$$

where $c_{k, l}(\mathrm{Ni}+n)$ is the $l^{\text {th }}$ chip of the $n^{\text {th }}$ bit in the $i^{\text {th }}$ code sequence of user $k, \omega_{l}$ is the $l^{\text {th }}$ carrier frequency, $\theta_{k, l}$ is the random phase of the $l^{\text {th }}$ subcarrier of user $k$ and uniformly distributed over $[0,2 \pi]$, and $L$ is the number of subcarriers.

In the receiver of Fig. 1(b), a size $L$ FFT is applied to the input. The output of the FFT is then despread to generate each bit of the received code sequence. The $N$ regenerated bits compose one code sequence, and the regenerated code is the input of the matched filter bank to detect the transmitted symbol. The $N$ despread bits form a degenerated code sequence, which is correlated with each of the possible $M$ code sequences. The sequence that gives maximum correlation is then mapped back into an $M$-ary symbol. The use of this narrowband multicarrier scheme provides frequency diversity for multipath mitigation so that no RAKE receiver is required, and a greater percentage of the received energy is actually collected for detection.

\section{B. Analysis of the output of the matched filter}

In this section, the output of the matched filter is analyzed. Because the MC-MC-CDMA transmitted waveform consists of a large number of narrowband subcarriers, the channel model can be reasonably approximated as a frequency selective Rayleigh fading channel where each subcarrier experiences flat Rayleigh fading. The subchannels can be written as

$$
g_{k, l}(t)=\beta_{k, l}(t) e^{j \psi_{k, l}(t)}
$$

which is a complex Gaussian random variable with zero mean and variance $\sigma^{2}$, characterized by a Rayleigh distributed amplitude attenuation $\beta_{k, l}(t)$, and a phase shift $\psi_{k, l}(t)$. While in practice there would be some correlation between adjacent subchannels, it is assumed here that $g_{k, l}(t)$ are uncorrelated and identically distributed for different $k$ and $l$.

If there are $K$ active users, the received signal of the synchronous system is

$$
\begin{aligned}
r(t)=\sum_{i=-\infty}^{\infty} \sum_{k=1}^{K} \sum_{l=1}^{L} \sum_{n=0}^{N-1} \beta_{k, l}(t) S_{k, i}(n) h\left(t-n T_{c}-i T_{s}\right) \\
\quad \times c_{k, l}(N i+n) \cos \left(\omega_{l} t+\phi_{k, l}(t)\right)+n(t),
\end{aligned}
$$

where $\phi_{k, l}(t)=\theta_{k, l}+\psi_{k, l}(t)$ and $n(t)$ is the additive white Gaussian noise with zero mean and power spectral density $N_{0}$. The amplitude attenuation and phase shift is considered to be constant over the time interval $\left[0, T_{c}\right)$.

From Fig. 1 and (7), the matched filter output at the receiver can be derived. Assume that the user 1 is the desired user and the $0^{\text {th }}$ transmitted $M$-ary symbol of the desired user is $m$ which represents an $M$-ary data symbol $b_{1,0}$, then the $0^{\text {th }}$ output of the filter matched to the code $m$ of user 1 is

$$
U_{1, m}=\int_{0}^{T_{s}} x_{1}(t) \sum_{n=0}^{N-1} v_{m}(n) h\left(t-n T_{c}\right) d t,
$$

where the demodulated code sequence of user $1 x_{l}(t)$ is

$$
\begin{gathered}
x_{1}(t)=\sum_{j=0}^{N-1} \lambda_{1, j} h\left(t-j T_{c}\right), \\
\lambda_{1, j}=\frac{1}{T_{c}} \int_{j T c}^{(j+1) T_{c}} r(t) \sum_{q=1}^{L} c_{1, q}(j) \cos \left(\omega_{q} t+\phi_{1, q}(j)\right) \alpha_{1, q} d t .
\end{gathered}
$$

In this paper, we consider Equal Gain Combining (EGC), because of its simplicity as the receiver does not require the estimation of the channel gain factor. Thus, $\alpha_{l, q}=1$ for all $q$. As shown in the Appendix, the matched filter output (8) can be written as [6], [7], [9]

$$
U_{1, m}=D_{1, m}+I_{1, m}+J_{1, m}+\eta
$$

where $D_{l, m}$ is the desired signal for user $1, I_{l, m}$ is the same carrier interference from other users, $J_{l, m}$ is other carrier interference from other users, and $\eta$ is the AWGN term with variance $N_{0} L N / 4 T_{c}$. The desired signal $D_{l, m}$ is

$$
D_{1, m}=\frac{1}{2} \sum_{n=0}^{N-1} v_{m}(n) v_{b_{1,0}}(n) \sum_{q=1}^{L} \beta_{1, q}(0) \text {. }
$$

In (11), the same carrier interference term $I_{l, m}$ can be written as

$$
\begin{aligned}
I_{1, m}=\frac{1}{2} \sum_{k=2}^{K} \sum_{l=1}^{L} \sum_{n=0}^{N-1} \beta_{k, l}(0) v_{m}(n) v_{b_{k, 0}}(n) \\
\quad \times c_{k, l}(n) c_{1, l}(n) \cos \left(\phi_{k, l}(n)-\phi_{1, l}(n)\right),
\end{aligned}
$$

and other carrier interference term $J_{l, m}$ can be written as 


$$
\begin{aligned}
& P_{e, M \text {-orthogonal } \beta} \\
& \quad=1-\int_{-\infty}^{\infty}\left[P\left(u>U_{1, m} \mid U_{1,1}=u\right)\right]^{M-1} P_{U_{1,1}}(u) d u \\
& \quad=1-\frac{1}{\sqrt{2 \pi \operatorname{var}\left(U_{1,1}\right)}} \int_{-\infty}^{\infty}\left(\int_{-\infty}^{u} \frac{1}{\sqrt{2 \pi \operatorname{var}\left(U_{1, m}\right)}} \exp \left(-\frac{x^{2}}{2 \operatorname{var}\left(U_{1, m}\right)}\right) d x\right)^{M-1} \exp \left(-\frac{\left(u-E\left(U_{1,1}\right)\right)^{2}}{2 \operatorname{var}\left(U_{1,1}\right)}\right) d u, \quad \text { where } m \neq 1
\end{aligned}
$$

$$
\begin{aligned}
J_{1}=\frac{1}{2 T_{c}} \sum_{n=0}^{N-1} \sum_{k=2}^{K} \sum_{l=1}^{L} & \sum_{\substack{q=1 \\
q \neq l}}^{L} \beta_{k, l}(0) v_{m}(n) v_{b_{k, 0}}(n) c_{k, l}(n) c_{1, q}(n) \\
& \times \int_{0}^{T_{c}} \cos \left(\left(\omega_{l}-\omega_{q}\right) t+\phi_{k, l}(t)-\phi_{1, q}(t)\right) d t .
\end{aligned}
$$

As shown in Appendix, $I_{l, m}$ and $J_{l, m}$ have zero mean and variance

$$
\begin{gathered}
\operatorname{var}\left(I_{1, m}\right)=\frac{1}{4}(K-1) L N \sigma^{2}, \\
\operatorname{var}\left(J_{1, m}\right)=\frac{\sigma^{2} N(K-1)}{8 \pi^{2}} \sum_{\substack{l=1 \\
l}}^{L} \sum_{\substack{q=1 \\
q \neq l}}^{L} \frac{1}{(l-q)^{2}},
\end{gathered}
$$

respectively. Therefore, assuming that we know the transmitted code sequence, the mean and variance of $U_{l, m}$, the statistics of output of the filter matched to the transmitted code sequence at time 0 is as follows:

$$
E\left(U_{1, m}\right)=\frac{1}{2} \sum_{n=0}^{N-1} \sum_{l=1}^{L} \beta_{1, l}(0),
$$

and

$$
\begin{aligned}
\operatorname{var}\left(U_{1, m}\right)=\frac{1}{4}( & K-1) L N \sigma^{2} \\
& +\frac{\sigma^{2} N(K-1)}{8 \pi^{2}} \sum_{\substack{l=1 \\
l}}^{L} \sum_{\substack{q=1 \\
q \neq l}}^{L} \frac{1}{(l-q)^{2}}+\frac{N_{0} L N}{4 T_{c}} .
\end{aligned}
$$

We now separately derive the probability of error for orthogonal and non-orthogonal code sequences.

\section{Probability of symbol error for the case of an M-ary signal using an orthogonal code sequence $(M \geq 2)$}

If we use the Gaussian approximation for MAI and assume that we know all the subcarrier channels, the PDF of the output of the matched filter corresponding to the transmitted code sequence $m$ for the desired user 1 is

$$
P_{U_{1, m}}(x)=\frac{1}{\sqrt{2 \pi \operatorname{var}\left(U_{1, m}\right)}} \exp \left(-\frac{\left(x-E\left(U_{1, m}\right)\right)^{2}}{2 \operatorname{var}\left(U_{1, m}\right)}\right),
$$

where $E\left(U_{l, m}\right)$ and $\operatorname{var}\left(U_{l, m}\right)$ are shown in (17) and (18). For simplicity, we assumed that the transmitted code sequence $m$ is 1 . The symbol error probability for the case of an $M$-ary orthogonal code sequence conditioned on the collection of subcarrier channels $P_{e, M \text {-orthogonal } \beta}$ is

$$
\begin{aligned}
& P_{e, M-\text { orthogonal } \beta} \\
& \quad=1-\int_{-\infty}^{\infty} P\left(u>U_{1,2}, \cdots, u>U_{1, M} \mid U_{1,1}=u\right) P_{U_{1,1}}(u) d u,
\end{aligned}
$$

where $U_{1, m}$ is the output of the matched filter corresponding to the code sequence $m$ for user $i, m=1, \ldots, M$. Since the $\left\{U_{1, m}\right\}$ are statistically independent, the joint probability function $P(u$
$\left.>U_{1,2}, u>U_{1,3}, \ldots, u>U_{1, m} \mid U_{1,1}=u\right)$ can be a product of $M-1$ marginal probabilities as follows,

$$
P\left(u>U_{1, m} \mid U_{1,1}=u\right)=\int_{-\infty}^{u} P_{U_{1, m}}(x) d x,
$$

where the PDF of the output of the matched filter corresponding to the code sequence $m(i \neq 1)$ is

$$
P_{U_{1, m}}(x)=\frac{1}{\sqrt{2 \pi \operatorname{var}\left(U_{1, m}\right)}} \exp \left(-\frac{x^{2}}{2 \operatorname{var}\left(U_{1, m}\right)}\right) .
$$

These probabilities are all same for $m=1, \ldots, M$. Then, the symbol error probability for the case of an $M$-ary orthogonal code sequence conditioned on the collection of subcarrier channels can be obtained as (23). The symbol error probability can be evaluated by Monte Carlo integration over the channel realization $\left\{\beta_{k, l}\right\}$. The symbol error probability conditioned on the collection of subcarrier channels (23) is the same when any one of the other $M-1$ code sequence is transmitted. Since all the $M$ code sequence are equally likely, the symbol error probability given in (23) is the average probability of a symbol error conditioned on the collection of subcarrier channels.

For $M=2$, The symbol error probability conditioned on the collection of subcarrier channels can be simplified to

$$
P_{e, \text { Binary-orthogonal } \beta}=Q\left(\frac{E\left(U_{1,1}\right)}{\sqrt{2 \operatorname{var}\left(U_{1,1}\right)}}\right) .
$$

\section{Probability of symbol error for the case of an M-ary signal using non-orthogonal code sequences $(M \geq 2)$}

For an $M$-ary signal using non-orthogonal code sequences, the average symbol error probability conditioned on the collection of subcarrier channels $P_{e, M \mid \beta}$ can be expressed as

$$
P_{e, M \mid \beta}=\frac{1}{M} \sum_{m=1}^{M} P_{e, m \mid \beta},
$$

where $P_{e, M \mid \beta}$ is the probability of error conditioned on the collection of subcarrier channels for the code sequence $v_{m}$. The probability of error $P_{e, M \mid \beta}$ is upper-bounded as

$$
P_{e, m \mid \beta} \leq \sum_{\substack{s=1 \\ s \neq m}}^{M} P_{e, M=2 \mid \beta}\left(v_{s}, v_{m}\right),
$$

where $P_{e, M=2 \mid \beta}\left(v_{s}, v_{m}\right)$ is the probability of error conditioned on the collection of subcarrier channels for a binary communication system using two non-orthogonal code sequences $v_{s}$ and $v_{m}$. The binary error probability $P_{e, M=2 \mid \beta}\left(v_{s}, v_{m}\right)$ is

$$
P_{e, M=2 \mid \beta}\left(v_{s}, v_{m}\right)=Q\left(\frac{1}{2} \sqrt{\frac{d_{s m}^{2}}{\operatorname{var}\left(U_{1, m}\right)}}\right),
$$

where $d_{s m}^{2}=\left\|\hat{S}_{s}-\hat{S}_{m}\right\|^{2}$. From (9), (10), the two sequences $\hat{S}_{s}$ 
and $\hat{S}_{m}$ after passing through the channel and demodulator are

$$
\begin{aligned}
\hat{S}_{j}=\sum_{n=0}^{N-1}\left(\frac{1}{T_{c}} \int_{n T_{c}}^{(n+1) T_{c}} r(t) \sum_{q=1}^{L} c_{i, q}(\right. & (n) \\
& \left.\cos \left(\omega_{q} t+\phi_{i, q}(n)\right) \alpha_{i, q} d t\right) \\
\times & h\left(t-n T_{c}\right), \quad j \in\{s, m\},
\end{aligned}
$$

where $i$ is the user index, and $r(t)$ is the received signal. Thus, the symbol error probability conditioned on the collection of subcarrier channels for an $M$-ary non-orthogonal code sequence is upperbounded as [20]

$$
P_{e \mid \beta, M} \leq \frac{1}{M} \sum_{\substack{m=1 \\ s \neq m}}^{M} Q\left(\frac{1}{2} \sqrt{\frac{d_{s m}^{2}}{\operatorname{var}\left(U_{1, m}\right)}}\right),
$$

which can be evaluated by Monte Carlo integration over the channel realizations $\left\{\beta_{k, l}\right\}$.

As shown in (29), the symbol error probability for the case of the non-orthogonal code sequence depends on the distance between code sequences in the code sequence set, as would be expected.

\section{Performance Comparison for Multi-code Multi- CARRIER CDMA}

In this section, the numerical BER performance of MC-MC-CDMA is compared to competing systems, and some properties of MC-MC-CDMA are observed. For the MC-MC-CDMA system, the chosen parameters are $N=16$ for the length of the code sequence, $L=16$ for the number of subcarriers, and $M=2,4,8,16$ for the $M$-ary symbols. The frequency selective Rayleigh fading channel is considered for the simulation. We assume that the channel on each subcarrier can be considered as flat fading and the receiver has perfect channel knowledge to detect the transmitted signal.

Fig. 2 shows the BER performance of the MC-MC-CDMA system with various $M$, the MC-CDMA system [6], and the multi-code single-carrier CDMA (MC-SC-CDMA) system [4]. In order to fairly compare the performance of these systems which have different subcarrier channel bandwidths, the number of subcarriers in each system is fixed to make the total bandwidth equal for all three systems. For example, when the length of the code sequence $N=M=16$, the MC-MC-CDMA system transmits 16 bits within one symbol time (4 information bits). That means the MC-MC-CDMA system uses 4 times more bandwidth compared to an MC-CDMA system with the same data rate. Therefore, we use 16 subcarriers for the MC-MC-CDMA system and 64 subcarriers for the MC-CDMA system. For the MC-SC-CDMA system, the length of the code sequence is 256 . Moreover, all three systems have the same data rate. Since the length of the code sequence $N$ is 16 , the maximum supportable data rate is 4 bits/symbol. Thus, both multi-code CDMA and the MC-CDMA systems have the same information rate of $4 \mathrm{bits} / \mathrm{symbol}$. In this way, all three systems use the same total bandwidth and the same data rate in the simulation. As can be seen, the proposed MC-MC-CDMA system performs better than the MC-CDMA system. By using multicarrier modulation, the MC-MC-CDMA system also easily outperforms the MC-SC-CDMA system in a frequency selective fading channel. Due to the gain which comes from orthogonality between code sequences and frequency spread-

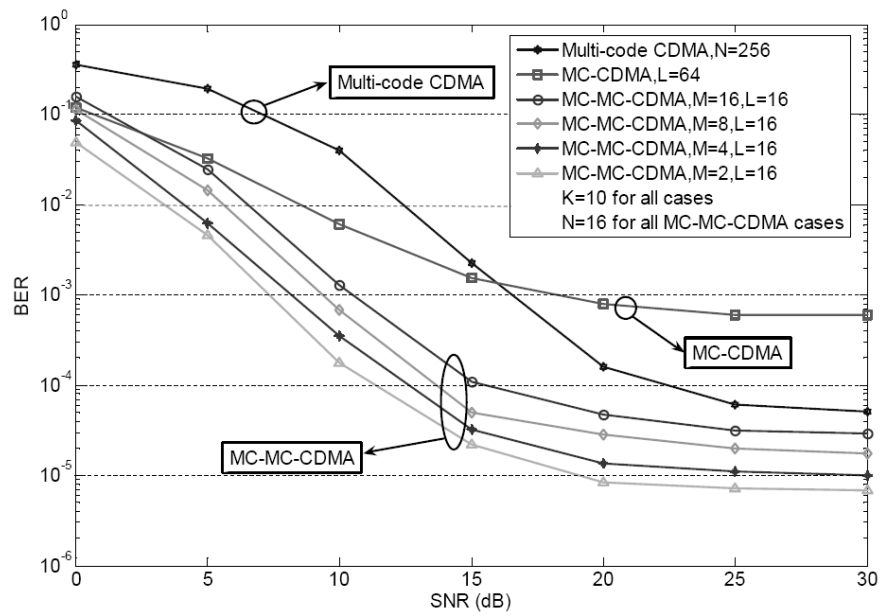

Fig. 2. Simulation results for BER versus SNR for MC-CDMA, MC-SC-CDMA, and MC-MC-CDMA with various $M$. All these systems occupy the same total bandwidth, and the MC-MC-CDMA system uses orthogonal code sequences since $M \leq N$.

ing gain, the proposed MC-MC-CDMA system shows better performance than MC-CDMA and MC-SC-CDMA systems. The performance can be adjusted to different channel conditions, since the time-frequency spreading tradeoff can be controlled accordingly.

In Fig. 3, the analytical expressions and the simulation results in a Rayleigh fading channel for the orthogonal code sequence case are compared. Here, $M=2$ and 16, and $K=10$. The performance of the $M=2$ case is better, because the 16-ary MC-MC-CDMA system uses more code sequences than the binary MC-MC-CDMA system. In the same $N=16$ dimensional signal space, it results in a smaller distance between code sequences than for the $M=2$ case. The plot shows that the analytical derivations agree closely with the simulation results for the orthogonal code sequence case.

Fig. 4 shows the analytical upperbound on symbol error probability and the simulation results for the MC-MC-CDMA system using non-orthogonal code sequences with various average code distances, as derived in Section III-D. Here, $M=$ $16, N=8, L=32$, and $K=10$. The code sequence set is randomly generated. In Fig. $4, d$ represents the average distance between code sequences:

$$
\begin{aligned}
d=\frac{1}{(M-1)(M-1)} \sum_{\substack{m=1 \\
s \neq 1 \\
s \neq m}}^{M}\left\|v_{m}-v_{s}\right\|, & \text { where } v_{i} \in \Omega, \quad i=1, \cdots, M .
\end{aligned}
$$

We notice that the simulation results fall in under the analytical upperbound, as expected. The upperbound is relatively tight. As shown in Section III-D, for the non-orthogonal code sequence, the symbol error probability upperbound depends on the distance between code sequences. Naturally, as the average distance between code sequences is decreased, the upperbound is increased.

The BER performance versus the number of users for both systems with an SNR of $10 \mathrm{~dB}$ is shown in Fig. 5. At the same BER, data rate per user, and consumed bandwidth, the MC-MC-CDMA system can support more users than the 


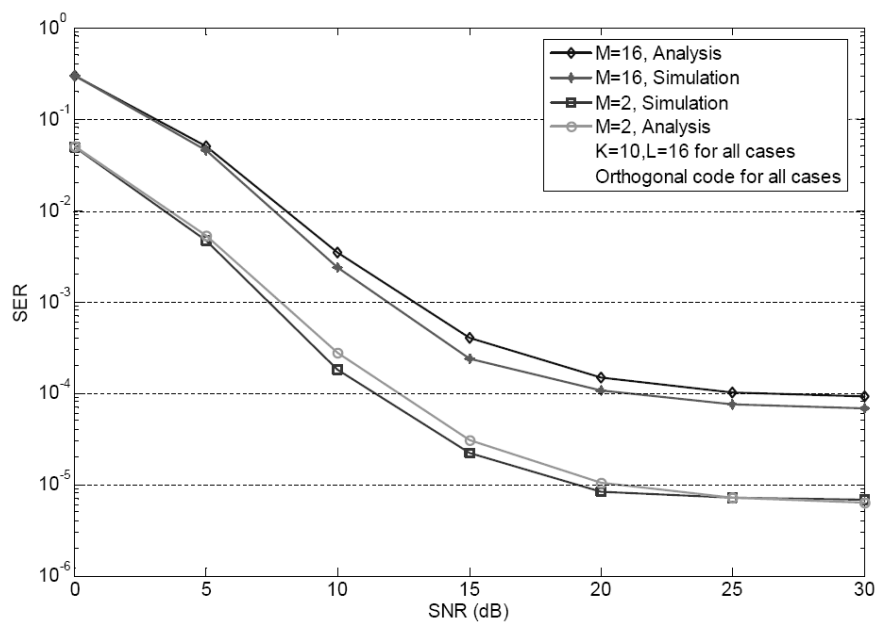

Fig. 3. The comparison of SER by analysis and SER by simulation for $M$-ary $(M=2, M=16)$ orthogonal code sequence cases in $L=16$ independent subcarrier channels

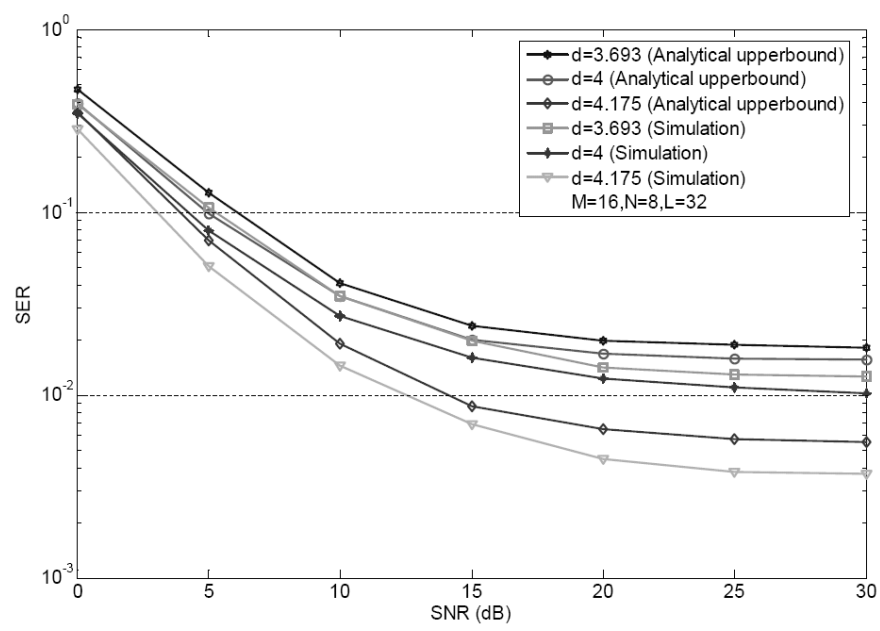

Fig. 4. The comparison of SER upperbound by analysis and SER by simulation for the MC-MC-CDMA system using non-orthogonal code sequence sets. $M=16, N=8, L=32, K=10$

MC-CDMA system. For example, at a BER of $3 \times 10^{-3}$, the number of users supported by the MC-MC-CDMA system is about 13, while it is about 7 for the MC-CDMA system. These are both uncoded systems with a total spreading gain of 64 .

Fig. 6 shows the received (pre-despreading) signal to interference plus noise ratio (SINR) versus $M$ with various numbers of users $K$ and SNR. In this system, the mean of all interference power is assumed to be equal. As shown in Fig. 6, the received SINR of the MC-MC-CDMA system varies according to the variation of $K$ and SNR, but not $M$. Since the length of the code sequence $N$ is fixed over all different value of $M$, the received SINR is not changed according to $M$ as shown in Fig. 6. It means that the proposed MC-MC-CDMA system can support higher data rate without increasing the interference unlike the multi-rate multicarrier CDMA system [17].

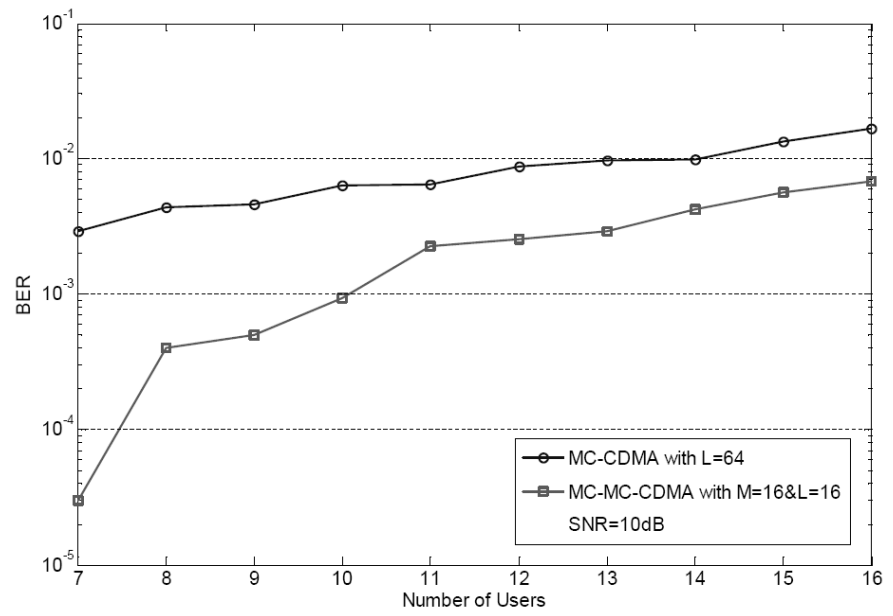

Fig. 5. The BER versus the number of users for the MC-CDMA system and the MC-MC-CDMA system. For the same total bandwidth, the MC-MC-CDMA can support a much higher system capacity than a conventional CDMA system.
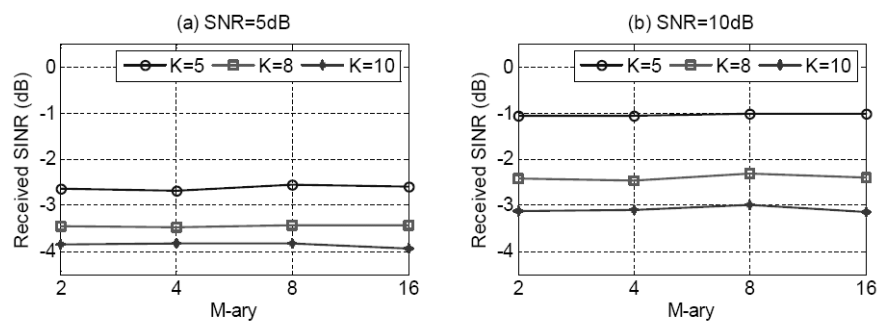

(c) $\mathrm{SNR}=15 \mathrm{~dB}$
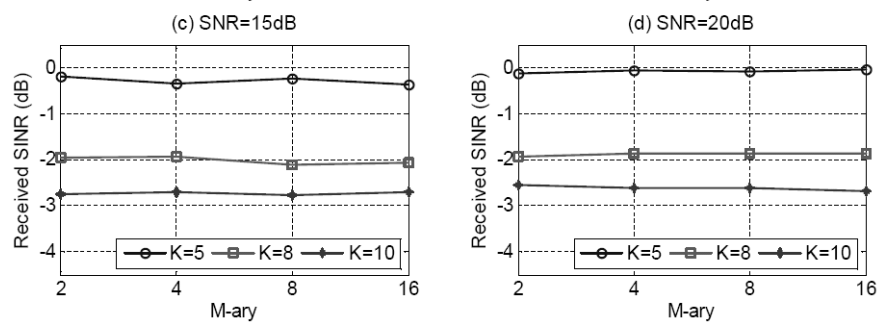

Fig. 6. The received (pre-despreading) SINR versus $M$ with various $K$ and SNR. It can be seen that the value of $M$ does not change the received SINR.

\section{CONCLUSION}

In this paper, multi-code multicarrier CDMA was shown to be a promising method for supporting variable data rates for a large number of users in future cellular systems. By using the multi-code concept, the MC-MC-CDMA system achieves two-dimensional gain as well as frequency diversity. In addition, various data rates can easily be supported by changing the size of the code sequence set. With the same total bandwidth, both analytical and simulation results showed that the proposed MC-MC-CDMA system clearly outperforms multicarrier CDMA and single carrier multi-code CDMA in terms of bit error probability and user capacity in a frequency selective Rayleigh fading channel. This shows that data rate flexibility can be achieved in a multicarrier CDMA system without any 
sacrifice in performance, and to the contrary, can actually allow improved robustness, flexibility, and capacity.

\section{APPENDIX}

\section{DECISION VARIABLE (THE OUTPUT OF THE MATCHED FILTER)}

For the analysis of the BER performance of the proposed system, the matched filter output can be written as (11). From (8) and (11), due to the orthogonality between subcarriers, the desired signal $D_{l, m}$ can be written as

$$
\begin{aligned}
D_{1, m}= & \frac{1}{T_{c}} \sum_{n=0}^{N-1} v_{m}(n) v_{b_{1,0}}(n) \int_{n T_{c}}^{(n+1) T_{c}} \sum_{q=1}^{L} \beta_{1, q}(n) \\
& \times h\left(t-n T_{c}\right) c_{1, q}^{2}(n) \cos ^{2}\left(\omega_{q} t+\phi_{1, q}(n)\right) d t \\
= & \frac{1}{2} \sum_{n=0}^{N-1} v_{m}(n) v_{b_{1,0}}(n) \sum_{q=1}^{L} \beta_{1, q}(n) .
\end{aligned}
$$

The interference term $I_{l, m}+J_{l, m}$ is given by

$$
\begin{aligned}
I_{1, m}+J_{1, m}= & \frac{1}{T_{c}} \sum_{j=0}^{N-1} v_{m}(j) \int_{j T_{c}}^{(j+1) T_{c}} \sum_{k=2}^{K} \sum_{l=1}^{L} \sum_{n=0}^{N-1} \beta_{k, l}(n) \\
& \times v_{b_{k, 0}}(n) h\left(t-n T_{c}\right) c_{k, l}(n) \cos \left(\omega_{l} t+\phi_{k, l}(n)\right) \\
& \times \sum_{q=1}^{L} c_{1, q}(j) \cos \left(\omega_{q} t+\phi_{1, q}(j)\right) d t,
\end{aligned}
$$

where $I_{1, m}$ corresponds to the interference from the other $K^{-1}$ users on the same subcarrier and $J_{l, m}$ corresponds to the interference from the other $K^{-1}$ users on the other subcarriers. Both $I_{1, m}$ and $J_{l, m}$ can be simplified as

$$
\begin{aligned}
& I_{1, m}=\frac{1}{T_{c}} \sum_{j=0}^{N-1} v_{m}(j) \sum_{k=2}^{K} \sum_{l=1}^{L} \sum_{n=0}^{N-1} \beta_{k, l}(n) v_{b_{k, 0}}(n) c_{k, l}(n) c_{1, l}(j) \\
& \times \int_{j T_{c}}^{(j+1) T_{c}} h\left(t-n T_{c}\right) \cos \left(\omega_{l} t+\phi_{k, l}(n)\right) \\
& \times \cos \left(\omega_{l} t+\phi_{1, l}(j)\right) d t \\
& =\frac{1}{2} \sum_{k=2}^{K} \sum_{l=1}^{L} \sum_{n=0}^{N-1} \beta_{k, l}(n) v_{m}(n) v_{b_{k, 0}}(n) \\
& \times c_{k, l}(n) c_{1, l}(n) \cos \left(\phi_{k, l}(n)-\phi_{1, l}(n)\right), \\
& J_{1, m}=\frac{1}{T_{c}} \sum_{j=0}^{N-1} v_{m}(j) \sum_{k=2}^{K} \sum_{n=0}^{N-1} \sum_{l=1}^{L} \sum_{\substack{q=1 \\
q \neq l}}^{L} \beta_{k, l}(n) v_{b_{k, 0}}(n) c_{k, l}(n) \mathcal{c}_{1, q}(j) \\
& \times \int_{j T_{c}}^{j T_{c}} h\left(t-n T_{c}\right) \cos \left(\omega_{l} t+\phi_{k, l}(n)\right) \\
& \times \cos \left(\omega_{q} t+\phi_{1, q}(j)\right) d t \\
& =\frac{1}{2 T_{c}} \sum_{k=2}^{K} \sum_{n=0}^{N-1} \sum_{l=1}^{L} \sum_{\substack{q=1 \\
q \neq l}}^{L} \beta_{k, l}(n) v_{m}(n) v_{b_{k, 0}}(n) c_{k, l}(n) c_{1, q}(n) \\
& \times \int_{0}^{T_{c}} \cos \left(\left(\omega_{l}-\omega_{q}\right) t+\phi_{k, l}(n)-\phi_{1, q}(n)\right) d t .
\end{aligned}
$$

As shown in (31)-(34), the matched filter output is expressed in terms of correlation functions of the code sequences. Now we can derive the variance of the term $I_{l, m}$ and $J_{l, m}$ for the EGC case. All cross terms are uncorrelated due to the random phase, and $I_{l, m}$ and $J_{l, m}$ are zero mean. Therefore, with the fact that $E\left[\beta_{k, l}^{2}\right]=2 \sigma^{2}$, the variance of $I_{l, m}$ and $J_{l, m}$ can be simplified as

$$
\begin{aligned}
\operatorname{var}\left[I_{1, m}\right] & \\
= & \frac{1}{4} \sum_{k=2}^{K} \sum_{l=1}^{L} \sum_{n=0}^{N-1} E\left[\beta_{k, l}^{2}(n)\right] E\left[v_{m}^{2}(n) v_{b_{k, 0}}^{2}(n) c_{k, l}^{2}(n) c_{1, l}^{2}(n)\right] \\
& \quad \times E\left[\cos ^{2}\left(\phi_{k, l}(n)-\phi_{1, l}(n)\right)\right] \\
= & \frac{1}{4}(K-1) L N \sigma^{2}, \\
\operatorname{var}\left[J_{1, m}\right] & \frac{1}{2 T_{c}^{2}} \sum_{k=2}^{K} \sum_{n=0}^{N-1} \sum_{l=1}^{L} \sum_{\substack{q=1 \\
q \neq l}}^{L} E\left[\beta_{k, l}^{2}(n)\right] \\
& \times E\left[v_{m}^{2}(n) v_{b_{k, 0}}^{2}(n) c_{k, l}^{2}(n) c_{1, q}^{2}(n)\right] \\
& \times E\left[\left(\int_{0}^{T_{c}} \cos \left(\left(\omega_{l}-\omega_{q}\right) t+\phi_{k, l}(n)-\phi_{1, q}(n)\right) d t\right)^{2}\right] \\
= & \frac{\sigma^{2}}{4 T_{c}^{2}} \sum_{k=2}^{K} \sum_{n=0}^{N-1} \sum_{l=1}^{L} \sum_{\substack{q=1 \\
q \neq l}}^{L} E\left[\left(\frac { T _ { c } } { 2 \pi ( l - q ) } \left\{\operatorname { s i n } \left(\left(\omega_{l}-\omega_{q}\right) t\right.\right.\right.\right. \\
& \left.\left.\left.\left.+\phi_{k, l}(n)-\phi_{1, q}(n)\right)-\sin \left(\phi_{k, l}(n)-\phi_{1, q}(n)\right)\right\}\right)^{2}\right] \\
= & \frac{\sigma^{2} N(K-1)}{8 \pi^{2}} \sum_{l=1}^{L} \sum_{\substack{q=1 \\
q \neq l}}^{L} \frac{1}{(l-q)^{2}} .
\end{aligned}
$$

\section{REFERENCES}

[1] T. S. Rappaport, Wireless communications, principles and practice, 2nd ed. Upper Saddle River, NJ: Prentice Hall PTR, 2002.

[2] C. L. I and R. D. Gitlin, "Multi-code CDMA wireless personal communications networks," IEEE International Conference on Communications, pp. 1060-1064, June 1995.

[3] C. L. I, G. P. Pollini, L. Ozarow, and R. D. Gitlin, "Performance of multi-code CDMA wireless personal communications networks," IEEE Vehicular Technology Conference, vol. 2, pp. 907-911, July 1995.

[4] H. D. Schotten, H. Elders-Boll, and A. Busboom, "Multi-code CDMA with variable sequence-sets," IEEE International Conference on Universal Personal Communications, pp. 628-631, October 1997.

[5] S. Hara and R. Prasad, "Overview of multicarrier CDMA," IEEE Communications Magazine, vol. 35, pp. 126-133, December 1997.

[6] X. Gui and T. S. Ng, "Performance of asynchronous orthogonal multicarrier CDMA system in a frequency selective fading channel," IEEE Transactions on Communications, vol. 47, no. 7, pp. 1084-1091, July 1999.

[7] E. A. Sourour and M. Makagawa, "Performance of orthogonal multicarrier CDMA in a multipath fading channel," IEEE Transactions on Communications, vol. 44, no. 3, pp. 356-367, March 1996.

[8] N. Yee, J-P. Linnartz and G. Fettweis, "Multi-carrier CDMA in indoor wireless radio networks," International Symposium on Personal, Indoor, and Mobile Radio Communications, pp. 109113, September 1993.

[9] J. G. Andrews and T. H. Meng, "Performance of multicarrier CDMA with successive interference cancellation in a multipath fading channel," IEEE Transactions on Communications, vol. 52, pp. 811-822, May 2004.

[10] L. L. Yang and L. Hanzo, "Multicarrier DS-CDMA: a multiple access scheme for ubiquitous broadband wireless communica- 
tions," IEEE Communications Magazine, vol. 41, pp. 116-124, October 2003.

[11] T. Ottosson and A. Svensson, "Multi-rate schemes in DS/CDMA systems," IEEE Vehicular Technology Conference, pp. 10061010, January 1995.

[12] U. Mitra, "Comparison of maximum-likelihood-based detection for two multi-rate access schemes for CDMA signals," IEEE Transactions on Communications, vol. 47, pp. 64-67, January 1999.

[13] 3GPP2, S. R0023, "High speed data enhancement for CDMA 2000 1xdata only," June 2000.

[14] "Technical overview of 1xEV-DV," White paper, Motorola Inc., September 2002, version G1.4. [Online]. Available: http://www.cdg.org

[15] P. Bender, P. Black, M. Grob, R. Padovani, N. Sindhushayana, and A. Viterbi, "CDMA/HDR: a bandwidth-efficient high-speed wireless data service for nomadic users," IEEE Communications Magazine, vol. 38, pp. 70-77, July 2000.

[16] H. D. Schotten, H. Elders-Boll, and A. Busboom, "Adaptive multi-rate multi-code CDMA systems," IEEE Vehicular Technology Conference, pp. 782-785, May 1998.

[17] P. W. Fu and K. C. Chen, "Multi-rate MC-DS-CDMA with multi user detections for wireless multimedia communications," IEEE Vehicular Technology Conference, vol. 3, pp. 1536-1540, May 2002.

[18] Y. W. Cao, C. C. Ko, and T. T. Tjhung, "A new multi-code/ multi-carrier DS-CDMA System," IEEE Global Telecommunications Conference, vol. 1, pp. 543-546, November 2001.

[19] P. W. Fu and K. C. Chen, "Multi-rate multi-carrier CDMA with multiuser detection for wireless multimedia communications," Wireless Communications and Networking Conference, vol. 1, pp. 385-390, March 2003.

[20] J. G. Proakis, Digital communications, 4th ed. New York, NY: McGraw-Hill, 2001.

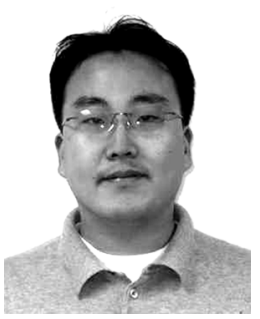

Taeyoon Kim is a Ph.D. candidate in the Department of Electrical and Computer Engineering at the University of Texas at Austin. He received B.S. and M.S. degrees in electrical engineering from Yonsei University, South Korea, in 1998 and 2000, respectively.

$\mathrm{He}$ was awarded the David Bruton, Jr. Graduate Fellowship for the 2004-2005 academic year by the Office of Graduate Studies at the University of Texas at Austin. He also received the fellowship from the South Korean Ministry of Information and Communication from 2002 to 2006. His research interests are in PHY of wireless communication systems, and are currently focused on the resource allocation in multiple-input multiple-output (MIMO) and multicarrier systems (OFDM, MC-CDMA) for the next generation wireless communications.

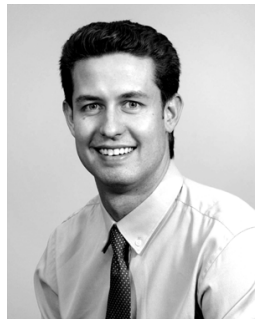

Jeffrey G. Andrews is an Assistant Professor in the Department of Electrical and Computer Engineering at the University of Texas at Austin, and Associate Director of the Wireless Networking and Communications Group (WNCG). He received the B.S. in Engineering with High Distinction from Harvey Mudd College in 1995, and the M.S. and Ph.D. in Electrical Engineering from Stanford University in 1999 and 2002, respectively.
He developed Code Division Multiple Access (CDMA) systems as an engineer at Qualcomm from 1995 to 1997, and has served as a frequent consultant on communication systems to numerous corporations, startups, and government agencies, including Microsoft, Palm, Ricoh, ADC, and NASA.

Dr. Andrews serves as an associate editor for the IEEE Transactions on Wireless Communications. He also is actively involved in IEEE conferences, serving on the organizing committee of the 2006 Communication Theory Workshop as well as regularly serving as a member of the technical program committees for ICC and Globecom. He is co-author of the forthcoming book from Prentice-Hall, Understanding WiMAX: Fundamentals of Wireless Broadband Networks. His research interests converge around multiuser wireless communications.

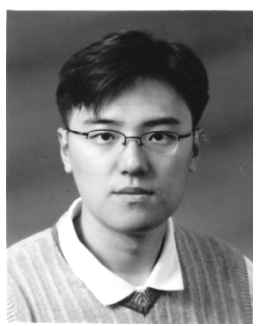

Jaeweon Kim is a Ph.D. student in the Department of Electrical and Computer Engineering at UT Austin. He received the B.S. and M.S. degrees from Korea Advanced Institute of Science and Technology (KAIST) in 1994 and 1996, respectively. He worked as a research engineer at the central $\mathrm{R} \& \mathrm{D}$ Center of SK Telecom, Inc. from 1996 to 2002, and as a research intern at Bandspeed Inc. since 2003. His current research interests focus on wireless communications, specially nonlinear signal processing of multicarrier systems for power efficient communcations.

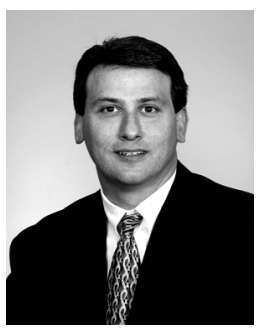

Theodore S. Rappaport is an active teacher, researcher, and entrepreneur. He has over 100 US and international patents issued or pending and has authored, co-authored, and coedited numerous books in the wireless field, including the popular textbooks Wireless Communications: Principles and Practice (Prentice-Hall, 1996, 2002), Smart Antennas for Wireless Communications: IS-95 and Third Generation CDMA Applications (Prentice Hall, 1999), and Principles of Communication Systems Simulation (Prentice Hall, 2004). He was recipient of the 1999 Stephen O. Rice Prize Paper Award from the IEEE Communications Society and the Stuart F. Meyer Award from the IEEE Vehicular Technology Society in 2005.

In 1989, Rappaport founded TSR Technologies, Inc., a cellular radio/PCS software radio equipment firm that he sold in 1993, and in 1995, he founded Wireless Valley Communications, Inc., a pioneering creator of software products for the design, measurement, and management of wireless networks. He is a registered professional engineer in the states of Virginia and Texas, and is a Fellow and past member of the board of directors of the Radio Club of America. Rappaport received the Marconi Young Scientist Award in 1990, an NSF Presidential Faculty Fellowship in 1992, and the Sarnoff Citation from the Radio Club of America in 2000. He received the James R. Evans Avant Garde award from the IEEE Vehicular Technology Society in 2002, the Frederick Emmons Terman Outstanding Electrical Engineering Educator Award from the ASEE in 2002, and the Outstanding Electrical and Computer Engineering Alumni Award from Purdue University in October 2004. Ted is married and has three children. 